\title{
Government Supervision Mode Selection in the Promotion Period of Prefabricated Construction Using Evolutionary Game
}

\author{
Shuwei Jing $(\mathbb{D}$, Zhuangyi Zhang $\mathbb{B}$, and Junai Yan \\ School of Management Science and Engineering, Shanxi University of Finance and Economics, Taiyuan, Shanxi, China \\ Correspondence should be addressed to Shuwei Jing; 20161034@sxufe.edu.cn
}

Received 22 February 2020; Revised 2 May 2020; Accepted 20 May 2020; Published 9 June 2020

Academic Editor: Chiara Bedon

Copyright (c) 2020 Shuwei Jing et al. This is an open access article distributed under the Creative Commons Attribution License, which permits unrestricted use, distribution, and reproduction in any medium, provided the original work is properly cited.

\begin{abstract}
Aiming at the speculative behavior of some developers who seek private interests in the promotion period of prefabricated construction, this research combines the actual situation, objectively and reasonably determines the parameters in the model, and builds an evolutionary game model to study the choice of government supervision mode in different situations, from the perspective of government supervision. The results showed that the choice of government supervision mode has great connection with the probability of identifying developers' speculative behavior when the government adopts node supervision. When the probability is greater than the developers' speculative value, the government will choose node supervision, while the developers will not adopt speculative behavior. Conversely, there will be a periodic behavior pattern in the evolutionary system, and the choice of government supervision mode is related to the value of each parameter. At the same time, the minimum probability of identifying speculative behavior that keeps the optimal situation stable is obtained. On this basis, the paper takes a practical case to discuss the influence of different parameter variations on the choice of government supervision mode and makes numerical simulations; then it puts forward some specific suggestions for government to restrain the speculative behavior of developer.
\end{abstract}

\section{Introduction}

Prefabricated construction refers to a building assembled on-site using prefabricated components produced by the factory. Compared with the traditional construction, it can effectively shorten the construction period, reduce construction waste, and save manpower. At the same time, with the disappearance of the demographic dividend and the requirement of environmental sustainability, the transformation of construction industry becomes very necessary in China. In recent years, China is actively developing prefabricated buildings to promote the transformation and upgrading of the construction industry. The government has promulgated the series of policies to guide the development of prefabricated buildings [1]. However, given that immature technology, lack of professionals, and the higher engineering cost currently, many developers who pursue private interests are unwilling to increase the prefabricated rate, which will seriously hinder further promotion of prefabricated construction. In order to reduce this phenomenon, the government has clearly stipulated the assembly rate of construction projects and issued a series of punitive measures to restrain. However, only by further strengthening supervision can government grasp the real implementation of prefabricated construction. Therefore, it is necessary to determine a reasonable government supervision mode to effectively promote prefabricated construction and ensure their quality and market environment.

At present, the promotion and supervision of prefabricated construction are widely studied. For the promotion of prefabricated construction, some scholars studied the adaptability factors of prefabricated construction, which mainly depend on labor shortage, housing demand, energy consumption, etc. [2-4]. Other authors combined the advantages of prefabricated construction in production efficiency and environmental protection to put forward the necessity of developing prefabricated construction [5-7]. In addition, some scholars have analyzed the public's concern about prefabricated buildings based on the technology acceptance theory, adopting web crawler technology combined 
with two methods of text mining. The results show that the public is relatively positive sentiments on prefabricated buildings, which is of great significance to the promotion of prefabricated buildings in China [8]. However, due to the deep-seated traditional building model, the promotion and development of prefabricated construction were not ideal. There were many factors that inhibit the development of prefabricated construction, for instance, lack of supply chain, inaccurate technical standards, and high capital cost. $[9,10]$. On this basis, a large number of scholars started to use quantitative methods such as principal component analysis (PCA) and gray DEMATEL analysis methods to derive the key factors that suppress the development of prefabricated construction and then further apply the AHP method to research the main inhibitory factor of capital cost [11-14]. In order to optimize the capital cost management, some scholars obtained that the site management and standardized design can greatly reduce the capital cost using the factor evaluation model $[15,16]$. In addition, from the perspective of developers, it was found that economic benefits, consumer demand, and policy incentives are the most important factors affecting the willingness of developers to develop prefabricated construction. Based on this, two incentives for developers to prefabricated construction were derived; the key paths of development were as follows: government incentive policy $\longrightarrow$ economic benefits $\longrightarrow$ development willingness, and consumer demand $\longrightarrow$ economic benefits $\longrightarrow$ development willingness [17].

For the supervision of prefabricated construction, there have been studies to summarize the research on prefabricated construction management from internationally renowned journals from 2000 to 2013 [18]. At the same time, government supervision is a key factor affecting the behavior of construction contractors. This has been confirmed by scholars through the use of SEM [19]. These studies have laid a solid foundation for futurity of research on assembled building supervision. Compared with traditional buildings, prefabricated construction is different in many aspects such as components and equipment; the focus of government supervision should also be different [20]. However, the present situation of prefabricated construction supervision in China is not adapted well to the characteristics of prefabricated construction. Research to build a modern information-based supervision system to achieve real-time monitoring of the whole process of prefabricated construction has become very popular. Existing research mainly developed a multidimensional Internet of Things- (IoT-) enabled BIM platform to achieve real-time visibility and traceability of prefabricated structures [21-24]. At the same time, the use of information systems must be effectively coordinated with government supervision to play a role. Government supervision issues focus on construction audits, dynamic monitoring of project data, and all-round interoperability of information systems. In order to effectively implement government supervision, optimization of information system based on the issues is necessary. Then, many scholars further studied the optimization of information systems and proposed a digital management system that combines the concept of BIM5D with radio frequency identification device technology [25-27], and based on this, the database of the above system is connected to the $\mathrm{C} \#$ language to achieve the effective integration of the two engineering technologies and the integration and visualization of the status information of the manufacturing components to provide guarantee for later quality monitoring [28].

In addition, there are two main methods to study the behavior choice of government supervision in academic circles. One is qualitative research; the final government behavior choice suggestion was proposed, through in-depth theoretical analysis of the specific environment in which the government was located [29]. However, this method was mainly used to study the unilateral behavior of the government. It lacks the influence of relevant stakeholders on the choice of government behavior and exists some subjectivities. Therefore, its application is not widespread. The other is quantitative research by applying game theory. Under the condition that the choice of government behavior was closely related to other stakeholders, the choice of government behavior was studied by constructing the game model of both parties. In this type of research, the behaviors of the government and the stakeholders were presented in a quantified form and the internal relationship between the behavioral choices of the two parties was considered in the actual situation, which is more objective and accurate, so it has been widely used by scholars [30-37]. At the same time, there is a small number of scholars who studied the government's behavior choices in market failure in macroeconomics by constructing simple models or revenue functions [38].

According to the above literature review, the supports of government policies and funds played an important role in the promotion and development of prefabricated construction. Meanwhile, research also showed that effective government supervision was a necessary means to promote the sustainable and high-quality development of prefabricated construction, and the discussion on real-time monitoring digital information platform was gradually deepening. Therefore, in order to effectively promote prefabricated construction and ensure sustainable development, it is necessary to study the supervision of prefabricated building projects by the government in China. However, the previous studies paid little attention to the choice of the supervision model of the government department and the issue of which supervision model is better in different situations during the period of vigorous promotion of prefabricated construction. In addition, the behavioral strategy choice between the government department and the developer meets the characteristics of the game, and the behavioral choices of the two parties are closely related, but they cannot find the optimal strategy in the initial state under the condition of limited rationality. Therefore, aiming at the speculative behavior of some developers in the promotion period of prefabricated construction, this paper will use evolutionary game method to analyze the strategic choice of government supervision modes.

The overall structure of this article is as follows. Section 1 presents the introduction and literature review, which is a 
major retrospective of prefabricated construction promotion and supervision and current research methods on government regulatory modes. Section 2 briefly introduces the theoretical basis of the evolutionary game model and describes the assumptions involved in the research process. Section 3 constructs the evolutionary game model that considers developers and government supervision departments and briefly analyzes the model results. Section 4 presents in depth the results of the model under different parameter values. Section 5 conducts numerical simulation to verify the reliability of the conclusion. Finally, conclusions, suggestions, and future research directions are presented in Section 6. The specific research ideas are shown in Figure 1.

\section{Basic Assumptions}

Evolutionary game theory is a theory that combines game theory analysis and dynamic evolution process analysis. It discards the assumptions of limited rationality and complete information on the basis of traditional game theory. From the perspective of system theory, it regards the adjustment process of group behavior as a dynamic system and incorporates the formation mechanism from individual behavior to group behavior and the various factors involved in it into the evolutionary game model to form a macromodel of microfoundation. Therefore, it can more truly reflect the diversity and complexity of actors and can provide a theoretical basis for macrocontrol group behavior [39].

During the promotion of prefabricated buildings, the behavioral strategy choices of government regulatory agencies and project developers conform to the characteristics of evolutionary games. Accordingly, this article proposes the following assumptions:

(1) There are two types of groups in the game model: developers and government supervision departments, both of which are of limited rationality and have limited information. Game members independently and dynamically choose their own behavioral strategies based on the benefits of the strategy.

(2) During the promotion of prefabricated construction, developers have two strategy choices: one is to build prefabricated construction projects as required in accordance with government policies and regulations of prefabricated construction, without speculation. The other is to adopt speculative behavior to make a profit, such as the fact that some construction processes are not completed in accordance with the policy requirements, reduce the prefabrication rate without authorization, etc. Therefore, the developer's strategy set is \{adopt speculative behavior, not adopt speculative behavior\}. Government supervision department also has two strategy choices. One is node supervision; that is, project deployment and result acceptance are performed only before and after the event. The other is to supervise the whole process of prefabricated construction, such as applying BIM technology or real-time monitoring based on digital information platforms, such as the Internet of

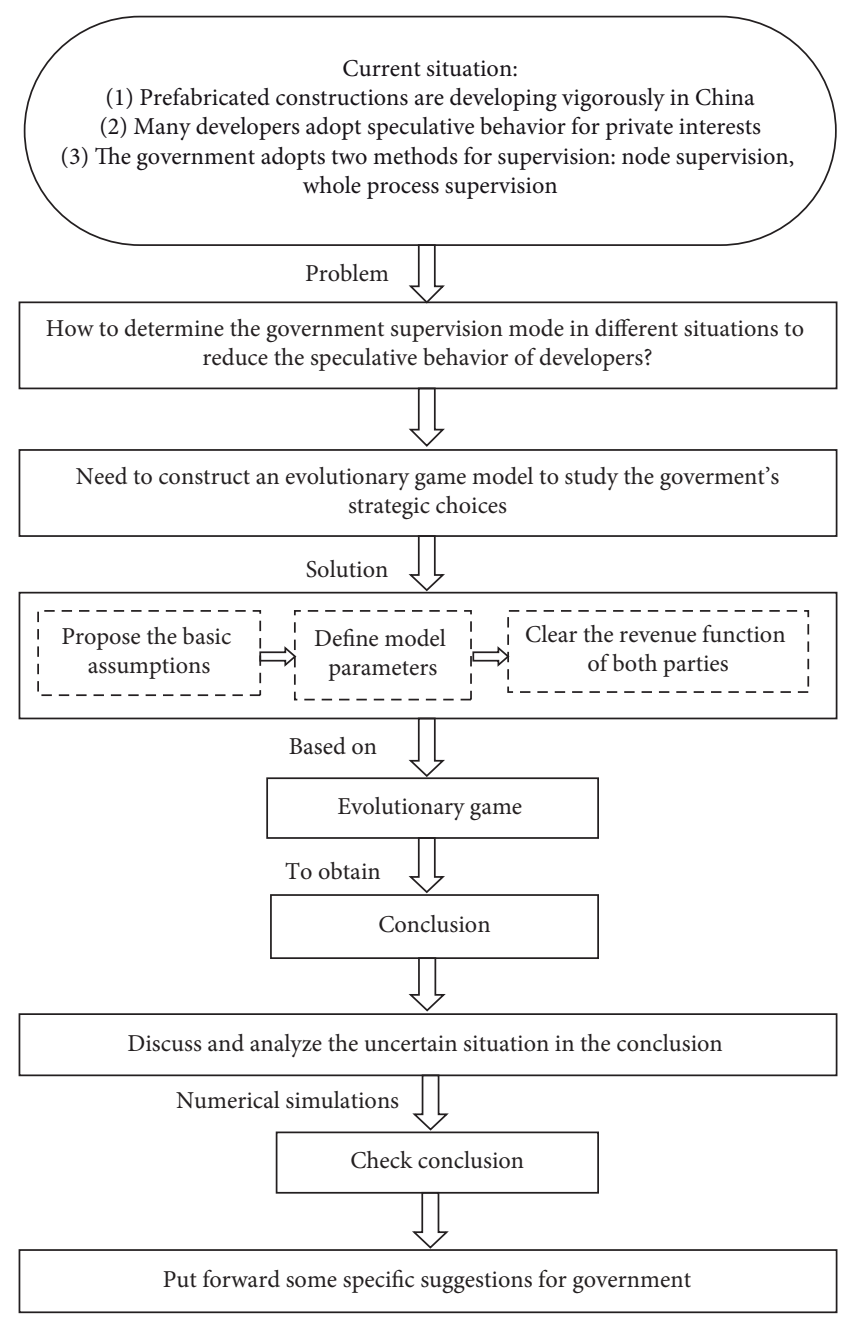

Figure 1: Research idea.

Things, and checking and accepting each key node. So, the strategy set of government supervision department is \{node supervision, whole process supervision\}.

(3) There is no collusion between the developer and the government, and neither party is affected by external environmental factors.

(4) When the government supervision department adopts the whole-process supervision, once the developer adopts speculative behavior, the supervision department can discover the behavior of the developer $100 \%$ in the first time. However, whether it is node supervision or whole-process supervision, as long as the speculative behavior of the developer is discovered, the supervision department will punish it.

(5) The regulatory actions of government departments will incur corresponding supervision costs, but the costs of the two supervision modes are different. The whole process supervision can be understood as an infinite number of node supervision activities within the time period of node supervision. Therefore, the whole process supervision cost is positively related to the result supervision cost. 


\section{Model Construction and Analysis}

3.1. Model Construction. Based on the previous assumptions, this paper analyzes the above-mentioned game groups and constructs the following game model, as shown in Table 1.

The meaning of each parameter is shown in Table 2.

In the promotion period of prefabricated construction, the motivation of developers to adopt speculative behavior is $R_{0}+\Delta R_{0}-C_{0} \leq R_{0}+\Delta R_{1}-C_{0}$; that is, $\Delta R_{1}-\Delta R_{0} \geq 0$, indicating that only when the net revenue from speculation is not less than nonspeculation, the developer may adopt speculative behavior. However, when developers adopt speculative behaviors, they also pay some price; that is, the government supervision departments fine them. Therefore, the "value" of developer's speculative behavior is $v=\left(\Delta R_{1}-\Delta R_{0} / P\right)$. In addition, the normal net revenue of developers without speculative behavior is $R_{0}+\Delta R_{0}-C_{0}$, but the minimum value of net revenue when they adopt speculative behavior is $R_{0}+\Delta R_{1}-C_{0}-P$. If the minimum value of the net revenue after the developers adopt speculative behavior and pay the penalty is still greater than the minimum value when no speculative behavior is taken, that is $R_{0}+\Delta R_{1}-C_{0}-P>R_{0}+\Delta R_{0}-C_{0}$, the developer will inevitably adopt speculative behavior. At this time, the functions of the supervision department are invalidated. Therefore, to ensure the effectiveness of the functions of the government supervision department, the punishment mechanism set-up must meet $P \geq \Delta R_{1}-\Delta R_{0}=\Delta R$. For the government supervision department, developers may adopt speculative behavior if they have speculative motivation. In the two government supervision modes, node supervision does not necessarily find developers' speculative behavior. Only when $\lambda_{1} S_{0}-\gamma C_{g}+P>\lambda_{2} S_{0}-C_{g}+\alpha P$, the government supervision department has the motivation to adopt the whole process supervision. Thus, the prerequisites for the selection evolutionary game problem discussed in this paper include $0 \leq \Delta R \leq P$ and $\lambda_{1} S_{0}-\gamma C_{g}+P>\lambda_{2} S_{0}-C_{g}+\alpha P$.

Assuming that during the promotion period of prefabricated construction $x$ refers to proportion of the members of the developers' group who do "not adopt speculative behavior," then $(1-x)$ is the proportion of members who "adopt speculative behavior." Meanwhile, the proportion of members in government supervision departments group who choose "whole process supervision "is $y$, and the proportion of members who choose "node supervision" is $(1-y)$.

For developers, the expected revenues of normal operation, speculation, and the whole developer group are as follows:

$$
\left.\begin{array}{rl}
U_{1 i} & =\left(R_{0}+\Delta R_{0}-C_{0}\right) y+\left(R_{0}+\Delta R_{0}-C_{0}\right)(1-y) \\
& =R_{0}+\Delta R_{0}-C_{0}, \\
U_{1 j} & =\left(R_{0}+\Delta R_{1}-C_{0}-P\right) y+\left(R_{0}+\Delta R_{1}-C_{0}-\alpha P\right)(1-y) \\
& =R_{0}+\Delta R_{1}-C_{0}-\alpha P-(1-\alpha) P y, \\
U_{1} & =x U_{1 i}+(1-x) U_{1 j} .
\end{array}\right\}
$$

For the government supervision departments, the expected revenues of whole process supervision $U_{2 i}$, node supervision $U_{2 j}$, and the whole government supervision department $U_{2}$ are as follows:

$$
\left.\begin{array}{rl}
U_{2 i} & =\left(\mathrm{S}_{0}-\gamma C_{g}\right) x+\left(\lambda_{1} \mathrm{~S}_{0}-\gamma C_{g}+P\right)(1-x) \\
& =\lambda_{1} \mathrm{~S}_{0}-\gamma C_{g}+P+\left(\mathrm{S}_{0}-\lambda_{1} \mathrm{~S}_{0}-P\right) x, \\
U_{2 j} & =\left(\mathrm{S}_{0}-C_{g}\right) x+\left(\lambda_{2} \mathrm{~S}_{0}-C_{g}+\alpha P\right)(1-x) \\
& =\lambda_{2} \mathrm{~S}_{0}-C_{g}+\alpha P+\left(\mathrm{S}_{0}-\lambda_{2} \mathrm{~S}_{0}-\alpha P\right) x, \\
U_{2} & =y U_{2 i}+(1-y) U_{2 j} .
\end{array}\right\}
$$

3.2. Model Analysis. According to equations (1) and (2), during the promotion period of prefabricated construction, the replicator dynamic equations of developers' choices of "not adopt speculative behavior" and government supervision department' choices of "whole process supervision" are as follows:

$$
\begin{aligned}
F_{1}(x, y) & =\frac{\mathrm{d} x}{\mathrm{~d} t}=x\left(U_{1 i}-U_{1}\right) \\
& =x(1-x)\left[\left(\alpha P+\Delta R_{0}-\Delta R_{1}\right)-(\alpha-1) P y\right] \\
F_{2}(x, y) & =\frac{\mathrm{d} y}{\mathrm{~d} t}=y\left(U_{2 i}-U_{2}\right) \\
& =y(1-y)\left[\left(\Lambda-\gamma C_{g}+C_{g}\right)-\Lambda x\right]
\end{aligned}
$$

where $\Lambda=\lambda_{1} S_{0}-\lambda_{2} S_{0}+P-\alpha P$.

When $\alpha=1$, the probability of identifying speculative behavior when the government adopts node supervision is $100 \%$; then the net revenue of developers who adopt speculative behavior is $R_{0}+\Delta R_{1}-C_{0}-P \leq R_{0}+\Delta R_{0}-C_{0}$. In addition, based on the principle of maximizing benefits, the government supervision department will choose the "node supervision" strategy and the final results of the strategy selection of both parties is "not adopt speculative behavior, node supervision."

When $0 \leq \alpha<1$, according to equations (3) and (4), the solution is

$$
\begin{aligned}
x^{*} & =\frac{\Lambda-\gamma C_{g}+C_{g}}{\Lambda}=1-\frac{\gamma C_{g}-C_{g}}{\Lambda} \\
& =\frac{\lambda_{1} S_{0}-\lambda_{2} S_{0}+P-\alpha P-\gamma C_{g}+C_{g}}{\lambda_{1} S_{0}-\lambda_{2} S_{0}+P-\alpha P}, \\
y^{*} & =\frac{\Delta R_{1}-\Delta R_{0}-\alpha P}{(1-\alpha) P} .
\end{aligned}
$$

As explained earlier, the prerequisites for the selection evolutionary game problem were discussed in this paper following as: $0 \leq \Delta R \leq P$ and $\lambda_{1} S_{0}-\gamma C_{g}+P>\lambda_{2} S_{0}-$ $C_{g}+\alpha P$. Meanwhile, the cost of the government to "adopt whole process supervision" is defined as greater than "adopt node supervision"; that is, $\gamma C_{g}>C_{g}$. According to the above conditions, when the probability $\alpha$ is in different intervals, the equilibrium points of the evolution system are shown in Table 3. 
TABLE 1: Game payoff matrix of developer and government supervision department.

\begin{tabular}{lcc}
\hline Developer & \multicolumn{2}{c}{ Government supervision department } \\
& Whole process supervision & Node supervision \\
\hline Not adopt speculative behavior & $R_{0}+\Delta R_{0}-C_{0}, S_{0}-\gamma C_{g}$ & $R_{0}+\Delta R_{0}-C_{0}, \mathrm{~S}_{0}-C_{g}$ \\
Adopt speculative behavior & $R_{0}+\Delta R_{1}-C_{0}-P, \lambda_{1} S_{0}-\gamma C_{q}+P$ & $R_{0}+\Delta R_{1}-C_{0}-\alpha P, \lambda_{2} S_{0}-C_{g}+P$ \\
\hline
\end{tabular}

TABle 2: Parameter specific meaning.

\begin{tabular}{|c|c|c|}
\hline Parameter & Meaning & Condition \\
\hline$R_{0}$ & Normal revenue for developers who do not adopt speculative behavior. & $R_{0} \geq 0$ \\
\hline$\Delta R_{0}$ & Government subsidies or rewards when developers do not adopt speculative behavior. & $0 \leq \Delta R_{0}<\Delta R_{1}$ \\
\hline$C_{0}$ & Capital cost of developers during normal development. & $C_{0} \geq 0$ \\
\hline$S_{0}$ & Social benefits brought by developers' normal development projects. & $S_{0} \geq 0$ \\
\hline$\Delta R_{1}$ & Additional profits for developers adopting speculative behavior. & $\Delta R_{1} \geq 0$ \\
\hline$P$ & Penalties for speculative behavior of developers. & $P \geq 0$ \\
\hline$C_{g}$ & Costs when the government adopts node supervision. & $C_{g} \geq 0$ \\
\hline$\gamma^{3}$ & Cost correlation coefficient for whole process supervision and node supervision. & $\gamma \geq 1$ \\
\hline$\lambda_{1}$ & $\begin{array}{c}\text { Social benefit coefficient of government department choosing whole process supervision when developer } \\
\text { adopts speculative behavior. }\end{array}$ & $0 \leq \lambda_{1} \leq 1$ \\
\hline$\lambda_{2}$ & $\begin{array}{c}\text { Social benefit coefficient of government choosing node supervision when developer adopts speculative } \\
\text { behavior. }\end{array}$ & $0 \leq \lambda_{2} \leq \lambda_{1} \leq 1$ \\
\hline$\alpha$ & Probability of identifying developers' speculative behavior when the government adopts node supervision. & $0 \leq \alpha \leq 1$ \\
\hline
\end{tabular}

TABLE 3: Stability of the evolution system of developers and government supervision departments.

\begin{tabular}{lc}
\hline Condition & Equilibrium points \\
\hline $0 \leq \alpha<\nu$ & $(0,0),(0,1),(1,0),(1,1),\left(x^{*}, y^{*}\right)$ \\
$\nu \leq \alpha<1$ & $(0,0),(0,1),(1,0),(1,1)$ \\
\hline
\end{tabular}

Then, the stability of the evolutionary system can be further calculated through the Jacobian matrix [40]. The Jacobian matrix is as follows:

$$
J=\left(\begin{array}{ll}
\frac{\partial F_{1}(x, y)}{\partial x} & \frac{\partial F_{1}(x, y)}{\partial y} \\
\frac{\partial F_{2}(x, y)}{\partial x} & \frac{\partial F_{2}(x, y)}{\partial y}
\end{array}\right)=\left(\begin{array}{cc}
a_{11} & a_{12} \\
a_{21} & a_{22}
\end{array}\right)
$$

where $a_{11}=(1-2 x)\left[\left(\alpha P+\Delta R_{0}-\Delta R_{1}\right)-(\alpha-1) P y\right], a_{12}=$ $x(1-x)(1-\alpha) P, \quad a_{21}=y(1-y)\left(\lambda_{2} S_{0}-\lambda_{1} S_{0}+\alpha P-P\right)$, $a_{22}=(1-2 y)\left[\left(\lambda_{1} S_{0}-\lambda_{2} S_{0}+P-\alpha P-\gamma C_{g}+C_{g}\right)-\left(\lambda_{1} S_{0}-\right.\right.$ $\left.\left.\lambda_{2} S_{0}+P-\alpha P\right) x\right]$.

Through calculation and stability analysis [41], the stability of equilibrium points of evolution system is finally obtained as shown in Table 4.

When $v \leq \alpha<1$, the probability of identifying developers' speculative behavior by government node supervision is greater than the "value" of developers' speculative behavior; there is an evolutionary stability strategy (ESS) of $(1,0)$ in the evolutionary game system between the government department and the developer, which means that the final strategy choice of both parties is "not adopt speculative behavior, node supervision." The results are shown in Figure 2.

When $0 \leq \alpha<\nu$, the probability of identifying developers' speculative behavior by government node supervision is less than the "value" of developers' speculative behavior; there is no evolutionary stability strategy (ESS) in the evolutionary game system between government departments and developers. The proportion of the developers who adopt speculative behavior is $1-x^{*}=\left(\gamma C_{g}-C_{g} / \Lambda\right)=$ $\left(\gamma C_{g}-C_{g} / \lambda_{1} S_{0}-\lambda_{2} S_{0}+P-\alpha P\right)$, while the proportion of the supervision departments who choose the whole process supervision is $y^{*}=\left(\Delta R_{1}-\Delta R_{0}-\alpha P /(1-\alpha) P\right)$, and the final evolutionary stability strategy is a hybrid strategy. The results are shown in Figure 3.

\section{Discussion and Analysis}

According to the analysis of the results, when $v \leq \alpha<1$, the final strategy choice of both parties is "not adopt speculative behavior, node supervision." However, when $0 \leq \alpha<\nu$, the evolutionary systems do not have the evolutionary stability strategy and forms a periodic behavior pattern. In the latter case, the final result of the evolution system has a great connection with the initial state of the system that is closely related to the size of each parameter. Therefore, the following analysis of different effects parameters on the initial state is to obtain the final results of the evolution of the system under different parameters.

4.1. The Influence of $\Delta R_{0}$ on Evolution System. When other parameters are determined, it can be easily obtained that $\Delta R_{0}$ has a negative correlation with the proportion $y^{*}$ of the government department's selection of the "whole process supervision" strategy during the prefabricated construction promotion period. It indicates that the greater the government's subsidy or reward $\Delta R_{0}$ for the developer's prefabricated construction, the greater the probability that the initial state will fall in areas I and II (as shown in Figure 4). That is, when the government grants more subsidies or rewards to developers for prefabricated construction, the 
TABLE 4: Stability analysis of each equilibrium point.

\begin{tabular}{|c|c|c|c|c|c|c|}
\hline Condition & Equilibrium point & $(0,0)$ & $(0,1)$ & $(1,0)$ & $(1,1)$ & $\left(x^{*}, y^{*}\right)$ \\
\hline \multirow{3}{*}{$0 \leq \alpha<v$} & $\operatorname{det} J$ & - & - & - & - & + \\
\hline & $\operatorname{trJ}$ & \pm & \pm & \pm & \pm & 0 \\
\hline & Local stability & Saddle point & Saddle point & Saddle point & Saddle point & Center point \\
\hline \multirow{3}{*}{$\nu \leq \alpha<1$} & $\operatorname{det} J$ & + & - & + & - & \\
\hline & $\operatorname{tr} J$ & + & \pm & - & \pm & \\
\hline & Local stability & Instability point & Saddle point & ESS & Saddle point & \\
\hline
\end{tabular}

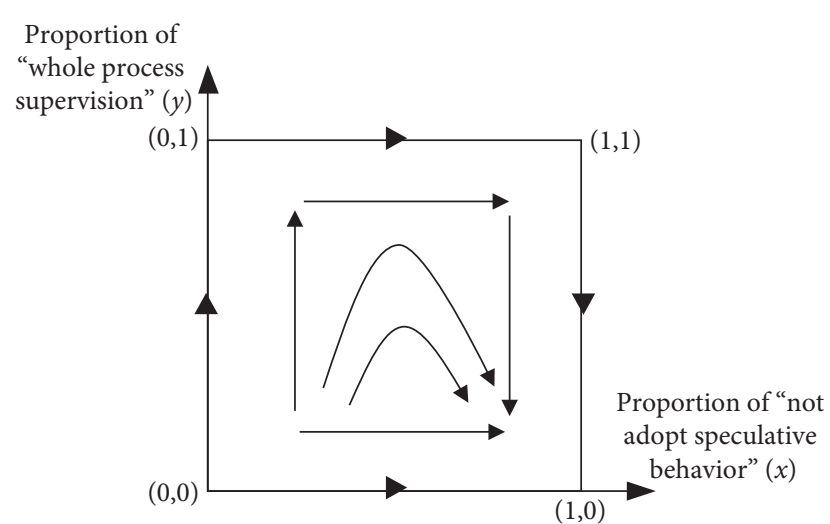

FIgURE 2: Phase diagram of dynamic evolution of equilibrium points $\nu \leq \alpha<1$.

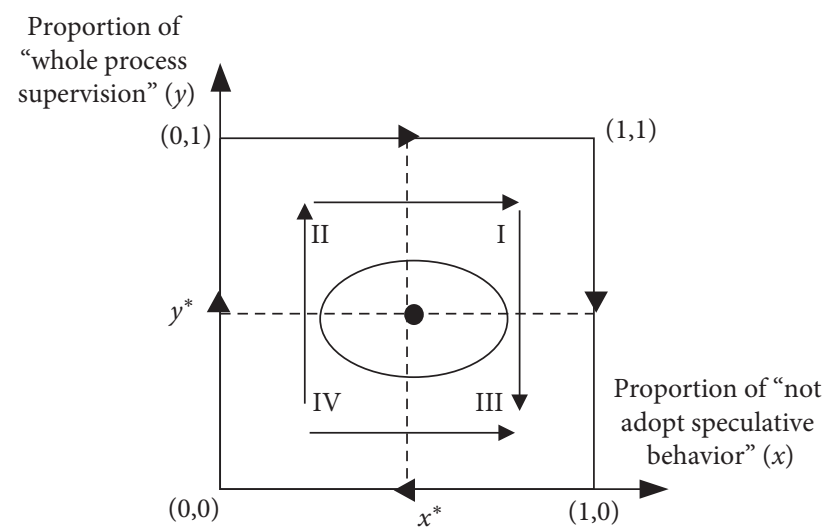

FIGURE 3: Phase diagram of dynamic evolution of equilibrium points $0 \leq \alpha<\nu$.

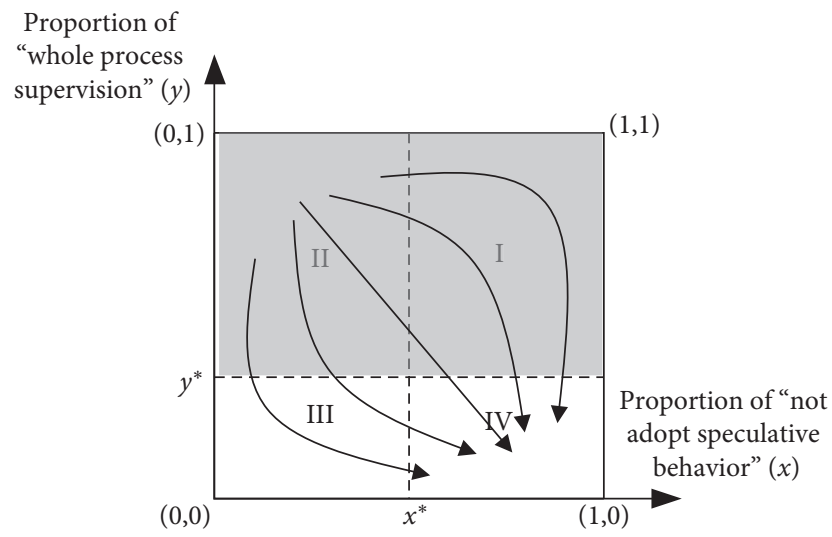

Figure 4: Phase diagram of dynamic evolution of equilibrium points $\left(\Delta R_{0}\right.$ increase $)$. temptation of speculative behavior to developers will decrease. Then more developers choose not adopt speculative behavior and government departments choose node supervision. Thus, the final result of the evolution system is "not adopt speculative behavior, node supervision."

4.2. The Influence of $\Delta R_{1}$ on Evolution System. When other parameters are determined, it can be easily obtained that $\Delta R_{1}$ has a positive correlation with the proportion $y^{*}$ of the government department's selection of the "whole process supervision" strategy during the prefabricated construction promotion period. It indicates that the large the profit obtained by the developer's speculative behavior, the greater the probability that the initial state will fall in areas III and IV (see Figure 5). That is, when developers make more profits from speculative behavior, the temptation of speculative behavior to developers will increase. Then more developers choose adopt speculative behavior and government departments choose whole process supervision. Thus, the final result of the evolution system is "adopt speculative behavior, whole process supervision."

4.3. The Influence of $\alpha$ on Evolution System. When other parameters are determined, it can be obtained that both the proportion $x^{*}$ of the developers choosing the strategy of "not adopt speculative behavior" and $y^{*}$ of the government departments choosing the strategy of "whole process supervision" have negative correlations with $\alpha$. It indicates that the greater the probability of identifying developers' speculative behavior when the government adopts node supervision, the greater the probability that the initial state will fall in area I (as shown in Figure 6). Thus, the final result of the evolution system is "adopt speculative behavior, node supervision."

4.4. The Influence of $\gamma$ on Evolution System. When other parameters are determined, it can be obtained that $\gamma$ has a negative correlation with the proportion $x^{*}$ that developers choose the strategy of "not adopt speculative behavior" during the promotion period of prefabricated construction. It dictates that the greater the cost correlation coefficient between the government "whole process supervision" and "node supervision"; that is, the higher the cost of the whole process supervision, the greater the probability that the initial state will fall in areas I and IV (as shown in Figure 7). When the cost of the whole process supervision increases, in order to save costs, the government departments choose 


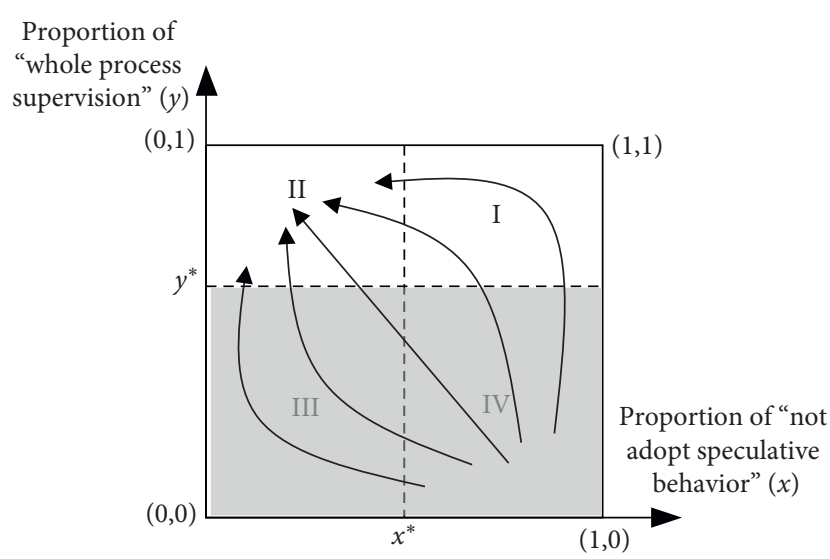

FIgURE 5: Phase diagram of dynamic evolution of equilibrium points $\left(\Delta R_{1}\right.$ increase).

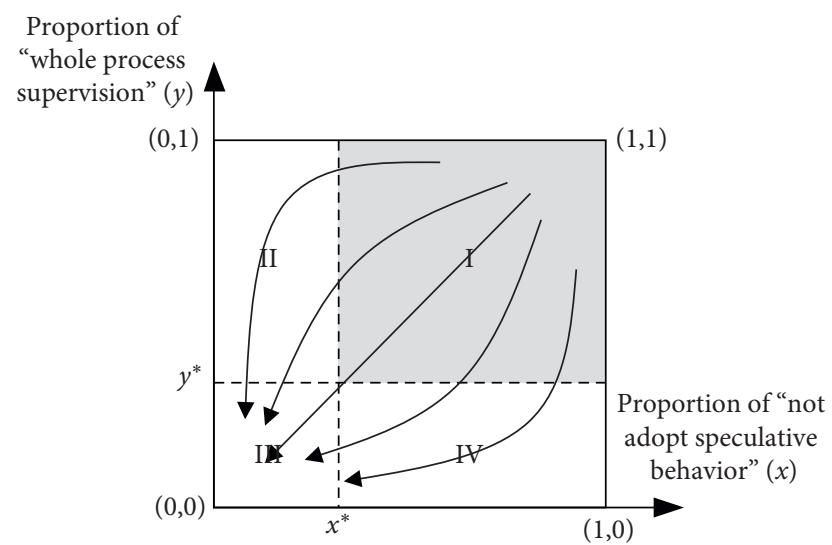

FIgURE 6: Phase diagram of dynamic evolution of equilibrium points ( $\alpha$ increase).

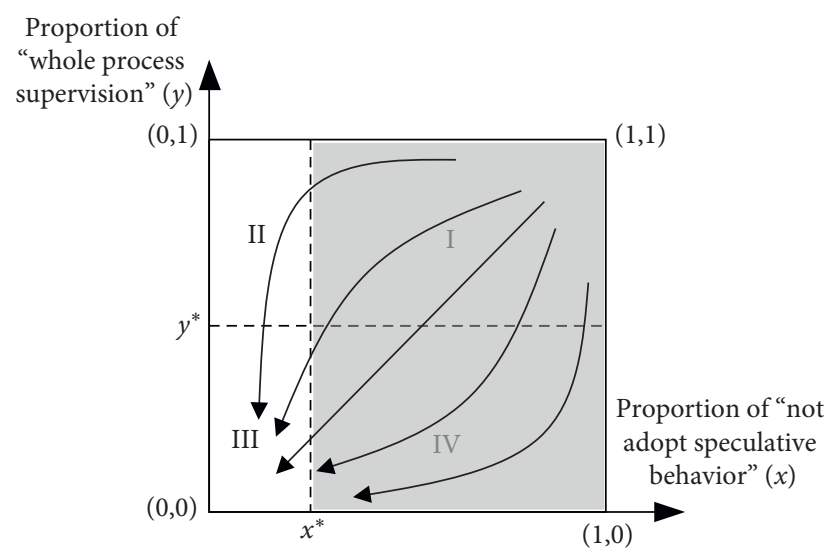

FIGURE 7: Phase diagram of dynamic evolution of equilibrium points ( $\gamma$ increase).

"node supervision," and the developers choose "adopt speculative behavior"; the final result of evolutionary system evolution is "adopt speculative behavior, node supervision."

4.5. The Influence of $P$ on Evolution System. When other parameters are determined, it can be obtained that $P$ has a positive correlation with the proportion of developers who choose the "no speculative behavior" strategy and a negative correlation with the proportion of government departments who choose the "whole process supervision" strategy. It dictates that the more the government fines the developer for speculative behavior, the greater the probability that the initial state will fall in area II (as shown in Figure 8). The final result of evolutionary system evolution is "not adopt speculative behavior, node supervision."

To conclude this section, when $0 \leq \alpha<\nu$, during the promotion period of prefabricated construction, the behavior choices of the developer and the government are affected by multiple factors. When there are more government subsidies or rewards, more penalties for developers who adopt speculative behavior, and higher process supervision costs, the government departments tend to choose the "node supervision" strategy. When the developers make more profits from speculative behavior, the developers tend to choose "adopt speculative behavior," and the government supervision departments choose the "whole process supervision" strategy.

\section{Numerical Simulations}

According to the analysis of the above results, when $v \leq \alpha<1$, the final strategy choice of both parties is "not adopt speculative behavior, node supervision." However, when $0 \leq \alpha<\nu$, the evolutionary system presents a periodic behavior pattern. This paper takes a prefabricated residential building project in Shanghai in recent years as an example and combines the local preferential and punishment policies for prefabricated construction to analyze these situations.

It is known that the normal revenue of the prefabricated construction project in the city is RMB 3.5 billion, and its development cost is RMB 1.90 billion. If the government supervision department adopts node supervision, the supervision cost is RMB 2 million, but if the whole process supervision is adopted, the supervision cost is RMB 3 million. If the developer meets the requirements, the government will give RMB 9 million in rewards to the project, but driven by the profits, if the developer adopts speculative behavior, it will receive an additional RMB 46 million in revenue. If the government supervision department chooses whole process supervision and discovers speculative behavior, it will impose a fine of RMB 60 million on the developer, and the social benefit is RMB 1.4 billion in this situation; but if the government chooses node supervision, the social benefit is only for RMB 1.2 billion. According to the above data, the initial conditions are selected: assuming that the probability that the developer chooses to take speculative behavior in the early stage of construction is 0.2 , and the probability that the government will adopt the entire process of supervision is 0.6 . Therefore, the parameters are selected as follows: $R_{0}=3500, \Delta R_{0}=9, C_{0}=1900, S_{0}=1800$, $\gamma=1.5 C_{g}=2, \Delta R_{1}=46, P=60, \lambda_{1}=0.78, \lambda_{2}=0.67, x_{0}=0.8$, $y_{0}=0.6, \nu=0.62$.

First of all, consider the situation of $v \leq \alpha<1$. When the probability of identifying developers' speculative behavior by government node supervision $=0.7$ (greater than 


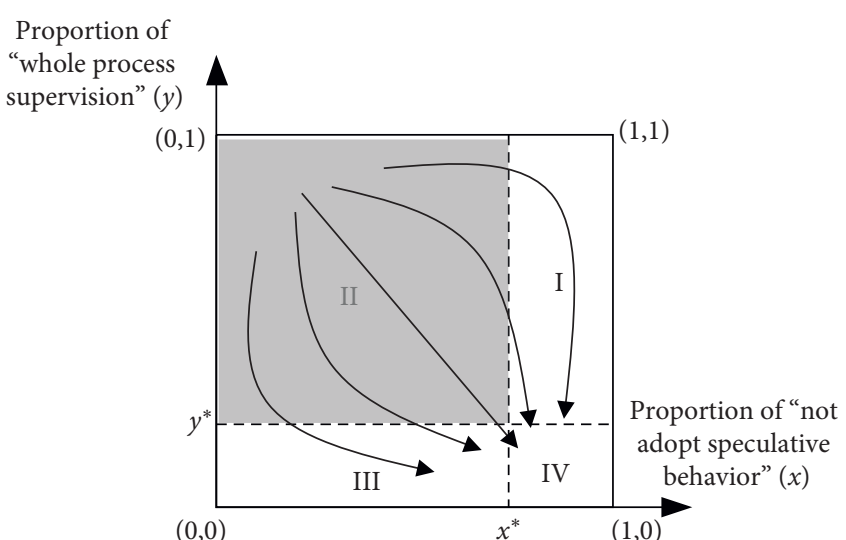

Figure 8: Phase diagram of dynamic evolution of equilibrium points ( $P$ increase).

developers' speculative value $=0.61$ ), the strategic choices between the government and developers are shown in Figure 9. From the figure, it can be seen that developers and the governments tend to choose "not speculative behavior, node supervision," which is a relatively ideal situation.

Secondly, analyze the situation of $0 \leq \alpha<v$, which is discussed in the two following types.

(1) On the one hand, when the probabilities of identifying developers' speculative behavior are $0.4,0.5$, and 0.6 (all less than developers' speculative value $v=0.61$ ), the fluctuation of the whole process supervision proportion selected by the government supervision department is shown in Figure 10. It can be seen that as the probability of identifying developers' speculative behavior by government node supervision increases gradually, the cycle of government supervision departments' whole process supervision gradually becomes longer, but the proportion of members who choose the whole process supervision decreases, and the government tends to choose node supervision. Conversely, the probability of identifying developers' speculative behavior by government node supervision decreases gradually, the cycle of government supervision departments' whole process supervision gradually becomes shorter, the proportion of government members who choose the whole process supervision gradually increases, and the time to discover developers' speculative behavior is longer. It can be concluded that the lower the probability of identifying speculative behavior by government node supervision is, the more it promotes the government to change to the whole process supervision mode which can quickly improve the supervision probability; the higher the probability of identifying speculative behavior by government node supervision, the more it promotes the government to change to the node supervision mode with low supervision cost.

(2) On the other hand, it can be seen from the above that strategic choice of government supervision department is also related to the "value" $v=\left(\Delta R_{1}-\Delta R_{0} / P\right)$

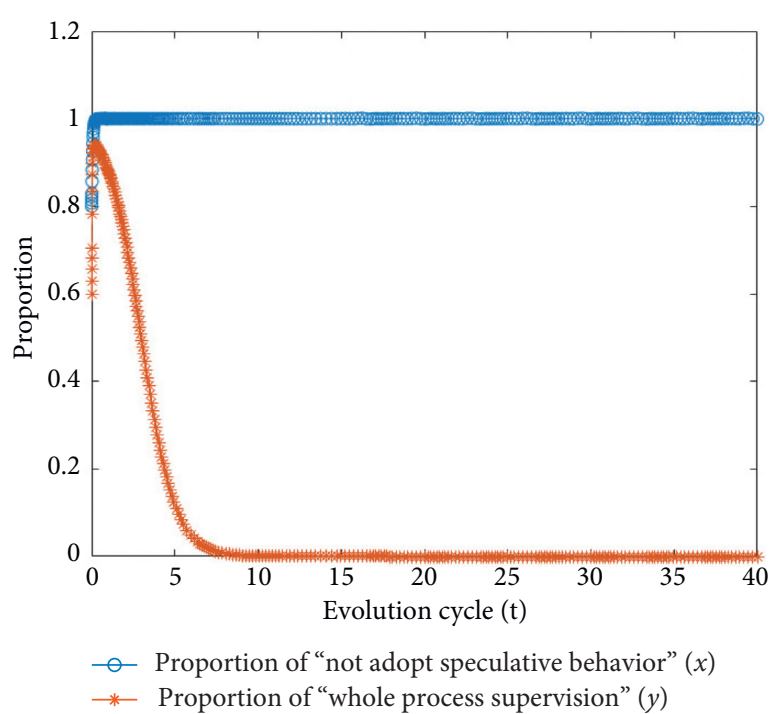

FIgURE 9: Evolution track of government departments and developers $(\nu \leq \alpha<1)$.

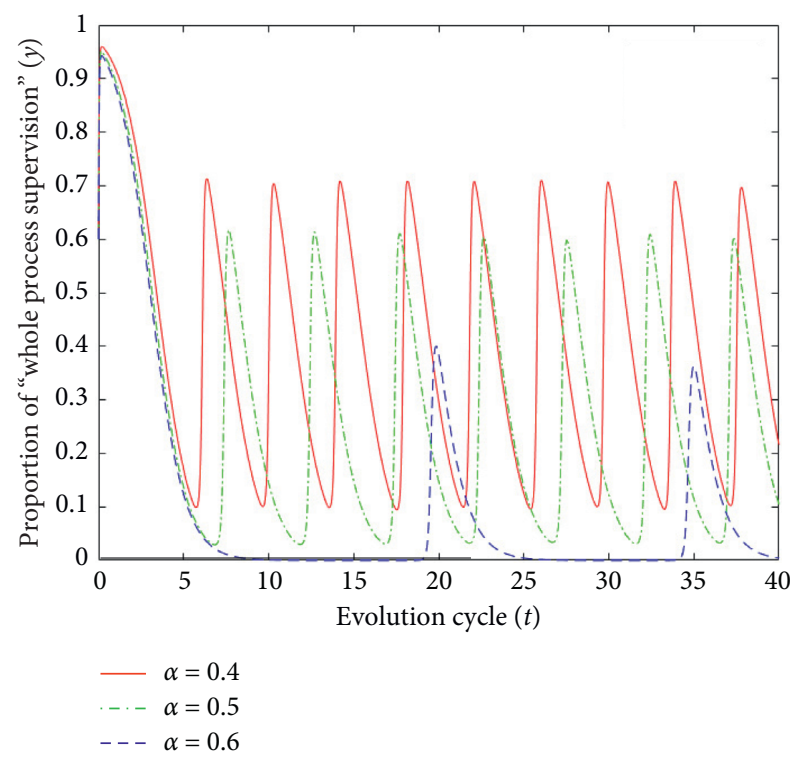

FIGURE 10: Evolutionary track with the change of $\alpha$.

of developers' speculative behavior. Assuming that the additional income obtained by the developer remains unchanged, the probability of identifying speculative behavior by government supervision nodes is still 0.5. It can be seen from Figure 11 that when the rewards set by the government supervision department remain unchanged and the penalties are RMB 60 million, RMB 55 million, and RMB 50 million, respectively-that is, when the "value" of developers' speculative behavior is $0.62,0.67$, and 0.74 , respectively-the cycle for the government supervision department to choose the whole process supervision gradually becomes shorter and the proportion of members to choose the whole process supervision gradually increases. In this evolutionary 


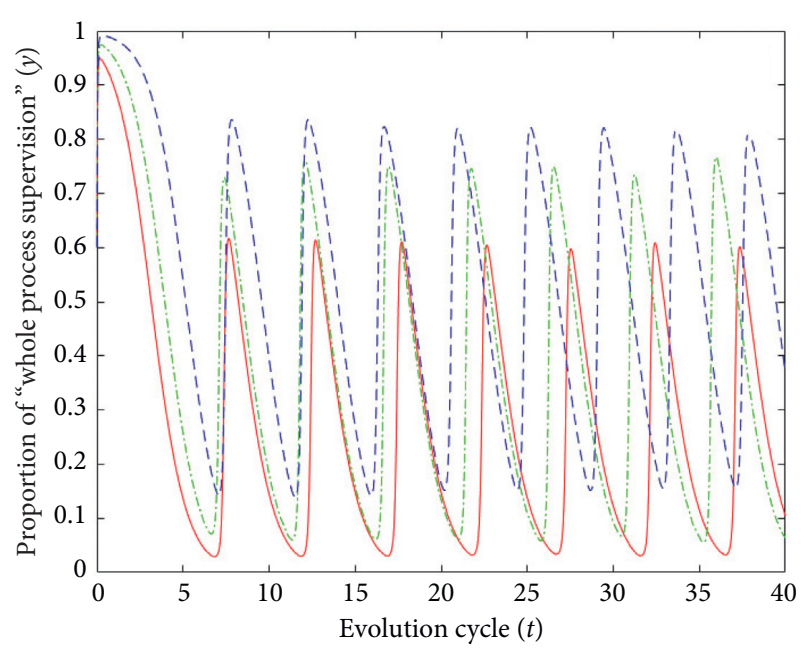

- $P=60$

-.. $P=55$

$---P=50$

Figure 11: Evolutionary track with the change of $P$.

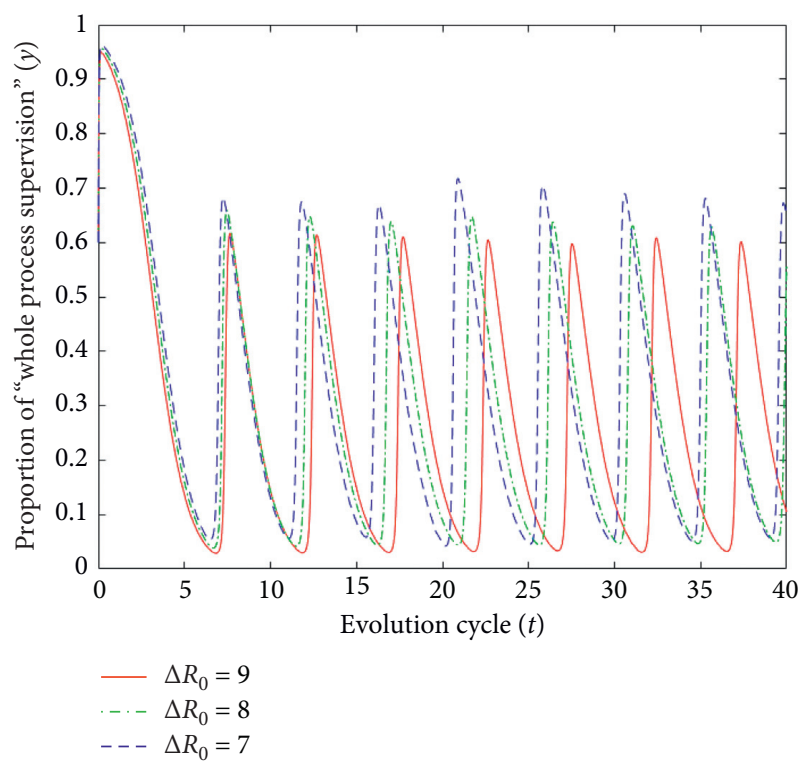

Figure 12: Evolutionary track with the change of $\Delta R_{0}$.

game system, the government is able to discover developers' speculative behavior in time for a longer period of time, thereby reducing developers' speculative behavior. In addition, it can be seen from Figure 12 that when the penalties set by the government supervision department remain unchanged, the rewards are RMB 9 million, RMB 8 million, and RMB 7 million, respectively; that is, when the "value" of developers' speculative behavior is $0.62,0.63$, and 0.65 respectively, the cycle for the government supervision department to choose the whole process supervision gradually becomes shorter. Similar to the above, developers will reduce speculative behavior. Therefore, for the government supervision department, its behavior choice has a great connection with the established reward and punishment mechanism. The fewer the penalties and the rewards, the higher the value of developers' speculative behavior and then the stronger the government's vigilance. It will promote government department to choose "whole process supervision" to improve the probability of identifying speculative behavior. On the contrary, the more the penalties and the rewards, the lower the value of developers' speculative behavior and then the weaker the government's vigilance. It will promote government department to choose "node supervision" to pursue low cost of supervision.

\section{Conclusions and Suggestions}

The results show that the choice of government supervision mode is closely related to the probability $\alpha$ of identifying developers' speculative behavior by government node supervision. When $\nu \leq \alpha<1$-that is, the probability of identifying speculative behavior by government node supervision is greater than the "value" of the behavior-there is an evolutionary stability strategy in the evolutionary game system, and the final choice of both parties is "not adopt speculative behavior, node supervision." When $0 \leq \alpha<\nu$-that is, the probability of identifying speculative behavior by government node supervision is less than the "value" of the behavior-there is no evolutionary stability strategy in the evolutionary game system, but a periodic behavior mode. Driven by their own interests, developers will choose speculative behavior based on the proportion of $1-x^{*}=\left(\gamma C_{g}-C_{g} / \Lambda\right)=\left(\gamma C_{g}-C_{g} / \lambda_{1} S_{0}-\lambda_{2} S_{0}+P-\alpha P\right)$, while government supervision department will choose the whole process of supervision based on the proportion of $y^{*}=\left(\Delta R_{1}-\Delta R_{0}-\alpha P /(1-\alpha) P\right)$, and the final evolutionary stability strategy is a hybrid strategy.

At the same time, combining the analysis of this article with the current actual situation in China, it can be inferred that the reason why the current research field is keen on studying the whole process supervision mode of information system. In addition to the role of information technology in the construction field, it is likely showing that the current government node supervision has a low probability of identifying developers' speculative behavior, and the value of developers' speculative behaviors is still at a high level. Therefore, if some measures can be taken to improve the probability of identifying speculative behavior by government node supervision, exceeding a certain threshold $v=\Delta R_{1}-\Delta R_{0} / P$, the strategic choices of both parties will automatically evolve to the optimal stable situation (not adopt speculative behavior, node supervision). At this time, the benefits of both parties have reached the maximum, and the prefabricated construction will be better popularized and developed. However, the limitation of this article is that it cannot accurately obtain the probability of identifying developers' speculative behavior when the government adopts node supervision, which leads to uncertainty about whether 
the current probability value has exceeded the current threshold. Therefore, further research is needed to determine the probability to formulate appropriate rewards and penalties and reduce effectively speculative behavior. Based on this, this paper makes the following recommendations:

(1) Increasing government funding and rewards for prefabricated construction projects appropriately: the reason why developers are not enthusiastic about investing in prefabricated construction is closely related to their high construction costs. Therefore, the appropriate increase in by the government will stimulate developers to invest in prefabricated construction. For example, the government can provide policy support for developers of prefabricated construction, such as giving priority to lending, increasing the amount and duration of loans, or directly granting government subsidies or reducing taxes on project developers that meet certain requirements for prefabrication rate.

(2) Striving to reduce the supervision cost of government department: on one hand, optimizing the internal structure of government supervision department to avoid overstaffing and actively adopting new and mature regulatory methods to reduce the cost of supervision technology and on the other hand, giving full play to the role of mass supervision and media supervision, providing financial rewards and government protection for whistleblower, and stimulating the supervision consciousness and enthusiasm of the masses and the media.

(3) Increasing the probability of identifying developers' speculative behavior: compared with the whole process supervision of prefabricated construction, the cost of node supervision is lower. Therefore, in order to increase the probability of identifying speculative behaviors in node supervision, during the promotion of prefabricated construction, the government should strengthen communication and contact developers, such as participating in major decision-making meetings of project companies to ensure the smooth development of the prefabricated construction project. At the same time, in the construction process, professionals can also be invited to accompany the site inspections to ensure the quality requirements of the prefabricated construction development process.

(4) Increasing the government's punishment for developers who fail to comply with the requirements for prefabricated construction: the effective punishment mechanism will restrain the speculative psychology of individual developers and play a role of deterring the whole so as to reduce the occurrence of speculative behavior. Therefore, it is necessary for the government to strengthen penalties for developers who adopt speculative behavior, such as increasing the amount of fine, perfecting relevant laws and regulations, and taking corresponding sanctions against developers who do not meet the requirements.

\section{Data Availability}

The data used to support the findings of this study are included within the article.

\section{Conflicts of Interest}

The authors declare that there are no conflicts of interest regarding the publication of this manuscript.

\section{Acknowledgments}

This research was supported by the Program for the Philosophy and Social Sciences Research of Higher Learning Institutions of Shanxi (nos. 2016241 and 2017326).

\section{References}

[1] G. W. Liu, Y. Tao, C. Mao, and P. P. Xu, "Textual and quantitative research on Chinese prefabricated construction policy from the perspective of policy tools," Journal of Chongqing University (Social Science Edition), vol. 24, no. 5, pp. 56-65, 2018.

[2] M. Arif and C. Egbu, "Making a case for offsite construction in China," Engineering, Construction and Architectural Management, vol. 17 , no. 6, pp. 536-548, 2010.

[3] K. M. A. El-Abidi and F. E. M. Ghazali, "Motivations and limitations of prefabricated building: an overview," Applied Mechanics and Materials, vol. 802, pp. 668-675, 2015.

[4] F. P. Rahimian, J. Goulding, A. Akintoye, and S. Kolo, "Review of motivations, success factors, and barriers to the adoption of offsite manufacturing in Nigeria," Procedia Engineering, vol. 196, pp. 512-519, 2017.

[5] D. O’Neill and S. Organ, "A literature review of the evolution of British prefabricated low-rise housing," Structural Survey, vol. 34, no. 2, pp. 191-214, 2016.

[6] K. M. A. El-Abidi, G. Ofori, and S. A. S. Zakaria, "Using prefabricated building to address housing needs in Libya: a study based on local expert perspectives," Arabian Journal for Science and Engineering, vol. 9, no. 44, pp. 8289-8304, 2019.

[7] L. F. Yu and J. C. Zhang, "Computer integrated construction system based on BIM," Journal of Civil Engineering and Management, vol. 32, no. 4, pp. 73-78, 2015.

[8] Y. Wang, H. Li, and Z. Z. Wu, "Attitude of the Chinese public toward off-site construction: a text mining study," Journal of Cleaner Production, vol. 238, 2019.

[9] X. L. Zhai, R. Reed, and M. Anthony, "Factors impeding the offsite production of housing construction in China: an investigation of current practice," Construction Management and Economics, vol. 32, no. 1-2, pp. 40-52, 2014.

[10] C. Mao, L. Shen, L. Luo, and Z. Li, "Identification of risk factors influencing the implementation of industrialized building system in China," in Proceedings of the 19th International Symposium on Advancement of Construction Management and Real Estate, pp. 219-230, Springer, Berlin, Germany, 2015.

[11] L. Jiang, Z. Li, L. Li, and Y. Gao, "Constraints on the promotion of prefabricated construction in China," Sustainability, vol. 10, no. 7, pp. 2516-2532, 2018.

[12] C. Mao, G. Liu, L. Shen, X. Wang, and J. Wang, "Structural equation modeling to analyze the critical driving factors and paths for off-site construction in China," KSCE Journal of Civil Engineering, vol. 22, no. 8, pp. 2678-2690, 2018. 
[13] Y. Han and L. Wang, "Identifying barriers to off-site construction using grey DEMATEL approach: case of China," Journal of Civil Engineering and Management, vol. 24, no. 5, pp. 364-377, 2018.

[14] H. J. Jia and Y. P. Xu, "Research on cost management of assembly building based on AHP," Construction Economy, vol. 39, no. 7, pp. 80-84, 2018.

[15] H. Xue, S. Zhang, Y. Su, and Z. Wu, "Factors affecting the capital cost of prefabrication-A case study of China," Sustainability, vol. 9, no. 9, pp. 1512-1533, 2017.

[16] H. Xue, S. J. Zhang, Y. K. Su, and Z. Z. Wu, "Capital cost optimization for prefabrication: a factor analysis evaluation model," Sustainability, vol. 10, no. 1, pp. 159-180, 2018.

[17] X. J. Liu and D. D. Li, "Research on the influencing factors and mechanism of development intention of prefabricated building," Construction Economy, vol. 40, no. 7, pp. 53-57, 2019.

[18] Z. Li, G. Q. Shen, and X. Xue, "Critical review of the research on the management of prefabricated construction," Habitat International, vol. 43, no. 1, pp. 240-249, 2014.

[19] Z. Z. Wu, A. T. W. Yu, and L. Y. Shen, "Investigating the determinants of contractor's construction and demolition waste management behavior in Mainland China," Waste Management, vol. 60, pp. 290-300, 2016.

[20] M. Beerepoot and N. Beerepoot, "Government regulation as an impetus for innovation: evidence from energy performance regulation in the Dutch residential building sector," Energy Policy, vol. 35, no. 10, pp. 4812-4825, 2007.

[21] R. Y. Zhong, Y. Peng, F. Xue et al., "Prefabricated construction enabled by the Internet-of-Things," Automation in Construction, vol. 76, no. 1, pp. 59-70, 2017.

[22] J. H. Cao, F. R. Ji, B. Z. Xie, and Z. J. Wu, "BIM-based prefabricated construction quality management," Journal of Civil Engineering and Management, vol. 34, no. 3, pp. 108-113, 2017.

[23] C. Z. Li, F. Xue, X. Li, J. Hong, and G. Q. Shen, “An Internet of Things-enabled BIM platform for on-site assembly services in prefabricated construction," Automation in Construction, vol. 89, pp. 146-161, 2018.

[24] Z. H. Zhang, H. Sun, Y. Liu, W. D. Feng, and Y. Xiao, "Exploration and practice of information management in prefabricated buildings," Journal of Engineering Management, vol. 32, no. 3, pp. 47-52, 2018.

[25] C. Z. Li, R. Y. Zhong, F. Xue et al., "Integrating RFID and BIM technologies for mitigating risks and improving schedule performance of prefabricated house construction," Journal of Cleaner Production, vol. 165, pp. 1048-1062, 2017.

[26] H. Qi, J. Q. Sun, N. Wang, Y. Zhu, and Y. Zhang, "Research on construction management of BIM and RFID applied in assembly building project," Construction Technology, vol. 47, no. 7, pp. 4-6, 2018.

[27] C. Chen, L. C. M. Tang, and Y. Jin, "Development of 5D BIMbased management system for pre-fabricated construction in China," in Proceedings of the International Conference on Smart Infrastructure and Construction (ICSIC), pp. 215-219, Cambridge, UK, July 2019.

[28] G. Ma, J. Jiang, and S. Shang, "Visualization of component status information of prefabricated concrete building based on building information modeling and radio frequency identification: a case study in China," Advances in Civil Engineering, vol. 2019, Article ID 6870507, 13 pages, 2019.

[29] P. Ren, "Local governments' choice strategies and their effects in policy conflicts," Journal of Public Management, vol. 12, no. 1, pp. 34-45, 2015.
[30] H. Zhou, S. L. Chen, and Y. M. Cui, "Study of supervision mechanism of tourism market based on evolutionary game model," Journal of Systems Engineering, vol. 31, no. 5, pp. 618-624, 2016.

[31] C. Li, X. Li, and Y. Wang, "Evolutionary game analysis of the supervision behavior for public-private partnership projects with public participation," Mathematical Problems in Engineering, vol. 2016, Article ID 1760837, 8 pages, 2016.

[32] X. L. Li, "Evolution game analysis of public-private partnership projects regulatory with consideration of reputation," Journal of Systems Engineering, vol. 32, no. 2, pp. 199-206, 2017.

[33] G. C. Wu, Y. Chen, and S. S. Xi, "Multi-scenario evolutionary game analysis of local governments' behavior choice in Housing Regulation Policy," Operations Research and Management Science, vol. 28, no. 5, pp. 61-70, 2019.

[34] Y. Song and C. M. Zhang, "Local government heterogeneity and regional environmental cooperation-evolutionary game analysis based on Chinese decentralization," Chinese Journal of Management Science, vol. 28, no. 1, pp. 201-211, 2020.

[35] M. Zhang, H. Li, L. Z. Xue, and W. W. Wang, "Using threesided dynamic game model to study regional cooperative governance of haze pollution in China from a government heterogeneity perspective," The Science of the Total Environment, vol. 694, 2019.

[36] M. Zhang, H. Li, Y. Song, and C. Li, "Study on the heterogeneous government synergistic governance game of haze in China," Journal of Environmental Management, vol. 248, no. 1, p. 109318, 2019.

[37] M. Zhang and H. Li, "New evolutionary game model of the regional governance of haze pollution in China," Applied Mathematical Modelling, vol. 63, pp. 577-590, 2018.

[38] C. Xu and Z. Y. Wang, "The role paradox and behavior choice of township government in rural public goods supply," Journal of Xi'an Jiaotong University (Social Sciences), vol. 36, no. 4, pp. 65-72, 2016.

[39] Y. Y. Yi and H. M. Liu, "Evolutionary game theory in economic research," Business Economics and Administration, vol. 166, no. 8, pp. 8-13, 2005.

[40] D. Friedman, "Evolutionary games in economics," Econometrica, vol. 59, no. 3, pp. 637-666, 1991.

[41] Q. W. Sun, L. Lu, G. L. Yan, and H. A. Che, "Asymptotic stability of evolutionary equilibrium under imperfect knowledge," Systems Engineering, vol. 23, no. 7, pp. 11-16, 2003. 